Article

\title{
Novel Diketopyrrolopyrrole-Based $\pi$-Conjugated Molecules Synthesized Via One-Pot Direct Arylation Reaction
}

\author{
Hui Liu ${ }^{1}$, Xiao-Feng Zhang ${ }^{1}$, Jing-Zhao Cheng ${ }^{1}$, Ai-Guo Zhong ${ }^{2}$, He-Rui Wen ${ }^{1, *}$ and \\ Shi-Yong Liu $1, *$ (D) \\ 1 School of Metallurgical and Chemical Engineering, Jiangxi University of Science and Technology, \\ Ganzhou 341000, China; liuhui666753@163.com (H.L.); 13398445081@163.com (X.-F.Z.); \\ cjz19960724@163.com (J.-Z.C.) \\ 2 Department of Chemistry, Taizhou University, Taizhou 317000, China; xg2268@163.com \\ * Correspondence: wenherui63@163.com (H.-R.W.); chelsy@zju.edu.cn (S.-Y.L.)
}

Received: 11 April 2019; Accepted: 5 May 2019; Published: 7 May 2019

\begin{abstract}
Diketopyrrolopyrrole (DPP) is an important type of $\pi$-conjugated building block for high-performance organic electronic materials. DPP-based conjugated materials are usually synthesized via Suzuki, Stille, or Negishi cross-coupling reactions, which require organometallic precursors. In this paper, a series of novel phenyl-cored DPP molecules, including five meta-phenyl-cored molecules and four para-phenyl-cored molecules, have been synthesized in moderate to good yields, in a facile manner, through the Pd-catalyzed direct arylation of $\mathrm{C}-\mathrm{H}$ bonds, and their optoelectrical properties have been investigated in detail. All new molecules have been fully characterized by NMR, MALDI-TOF MS, elemental analysis, UV-visible spectroscopy, and cyclic voltammetry. This synthetic strategy has evident advantages of atom- and step-economy and low cost, compared with traditional cross-coupling reactions.
\end{abstract}

Keywords: diketopyrrolopyrrole (DPP); direct arylation; $\pi$-conjugated molecules; cyclic voltammetry; atom economy

\section{Introduction}

$\pi$-Conjugated materials play an irreplaceable role in the field of optics and organic electronics. $\pi$-Conjugated organic materials can be divided into polymers (molecular weight MW $>10,000$ ), oligomers (MW > 1000) and small molecules (MW < 1000) [1]. Since the discovery of polyacetylene in 1976 [2], $\pi$-conjugated polymers, oligomers, and small molecules have drawn extensive attention from researchers. Essentially, the polymers are mixtures of various polymeric chains with different MWs and polydispersity index (PDI) greater than 1. Although the small molecules have defined structures, their conjugation lengths and molecular weights are limited. Nevertheless, the oligomers can be regarded as a kind of special polymer with monodispersity $(\mathrm{PDI}=1)$ and defined structure which might combine the advantages of polymers and small molecules while overcoming their individual shortcomings $[3,4]$. Therefore, oligomers with defined structure and high molecular weight are expected to be ideal choices for organic $\pi$-conjugated functional materials.

Since the discovery of diketopyrrolopyrroles (DPPs) by Farnum [5] et al. in 1974, a large number of DPP-based conjugated molecules or polymers have been synthesized by researchers. Thienyl-flanked DPP (TDPP) [6] has become a popular building block, with a planar structure and being amenable to easy structural modifications [7]. The electron-rich thiophene can give rise to strong intramolecular charge transfer due to the electron-deficient DPP core and intermolecular $\pi-\pi$ stacking, which improves the optical and electrochemical properties. Small molecules based on DPP [8-11], core-modified with 
two thiophene rings, have also been explored in Organic Photovoltaic Cells (OPVs). Conjugated molecules based on TDPP can be simultaneously used as electron donors and acceptors with bipolar charge-transporting properties. The $\alpha-\mathrm{C}-\mathrm{H}$ bonds on thiophene rings can be directly arylated by $\mathrm{C}-\mathrm{X}$ ( $\mathrm{X}=\mathrm{Br}$ or I) under palladium catalysis [12-16], due to the ease of palladation through a concerted metalation-deprotonation (CMD) pathway [17], leading to activation of the $\mathrm{C}-\mathrm{H}$ bonds on $2 / 5$ positions on the thiophene rings.

DPP-based conjugated materials are usually synthesized by traditional cross-coupling reactions, such as Kumada [18], Suzuki [19], Stille [20,21], and Yamamoto [22], which usually involve multiple steps and pre-functionalization of substrates with $\mathrm{C}-\mathrm{M}$ bonds, a stoichiometric amount of $\mathrm{Ni}(0)$ catalyst, and low overall yields. Direct C-H arylation, as an atom- and step-economic synthetic strategy [23-32], will overcome these shortcomings and avoid the tedious synthetic steps with higher atomic economy and easier purification compared with the traditional C-C couplings [33].

In continuation of our interest in the atom-efficient synthesis of $\pi$-conjugated materials [30,32,33], in this study, we synthesized DPP-based molecules with A-D-A structures by using TDPP as the electron-deficient unit and phenyl derivative units as the electron-rich unit. Here, the $\alpha-\mathrm{C}-\mathrm{H}$ bonds on TDPP were directly arylated by the $\mathrm{C}-\mathrm{Br}$ bonds from phenyl bromides under palladium catalysis. All of the target complex molecules were constructed from simple starting reactants and in a single step. The whole synthetic process was atom- and step-economical. The structures of nine molecules were characterized and verified by NMR, MALDI-TOF MS, and elemental analysis, and their optical and electrochemical properties were investigated by UV-vis absorption and cyclic voltammetry (CV) spectroscopy.

\section{Results and Discussion}

Firstly, TDPP and dibromobenzene derivatives were chosen as two kinds of substrates for the direct $\mathrm{C}-\mathrm{H}$ arylation oligomerization. Here, the protocol of $1.5 \mathrm{~mol} \% \mathrm{Pd}_{2}(\mathrm{dba})_{3}$ as pre-catalyst, $3 \mathrm{~mol} \% \mathrm{P}(o-\mathrm{MeOPh})_{3}$ as ligand, $30 \mathrm{~mol} \% \mathrm{PivOH}$ as additive, 2 equiv $\mathrm{Cs}_{2} \mathrm{CO}_{3}$ as base, and toluene as solvent, which were previously carefully developed by our group [33], was applied to these direct $\mathrm{C}-\mathrm{H}$ arylated couplings. The carboxylic acid PivOH, employed herein, acted as a co-catalyst for the CMD activation of $\mathrm{C}-\mathrm{H}$ bonds [34,35]. As can be seen in Table 1, by controlling the reactant ratio of the 1:0.4 between TDPP and dibromobenzene derivatives [33], the optimized yields of $50 \% \sim 60 \%$ for the target molecules have been obtained. The $\alpha-\mathrm{C}-\mathrm{H}$ bonds on the starting TDPP were directly arylated by $m$-dibromobenzene derivatives, 1,3-dibromo-5-nitrobenzene, 1,3-dibromo-5-fluorobenzene, 1,3-dibromo-5-chlorobenzene, 1,3-dibromo-5-methoxy benzene, and 3,5-dibromo-1,1'-biphenyl, producing DPP-based molecules Ms1 5, respectively, with 50\%, 57\%, 53\%, $49 \%$, and $57 \%$ yields. The direct arylation coupling between DPP and the $p$-dibromobenzene derivatives, 1,4-dibromo-2,3-difluorobenzene, 1,4-dibromo-2,5-difluorobenzene, 1,4-dibromo-2,5-dichlorobenzene, and 1,4-dibromo-2,5-dimethoxy benzene, produced DPP-based molecules Ms6 9, respectively, with $50 \%, 61 \%, 56 \%$, and $60 \%$ yields.

All nine synthesized $\pi$-conjugated molecules have been characterized and verified by NMR, MALDI-TOF MS, and elemental analysis. The ${ }^{1} \mathrm{H}-\mathrm{NMR}$ analysis reveals spectra changes of molecules with phenyl cores bearing various groups, including electron-withdrawing groups such as $-\mathrm{NO}_{2},-\mathrm{F}$, and $-\mathrm{Cl}$, electron-neutral phenyl groups, and the electron-donating group - $\mathrm{OCH}_{3}$. The electron-withdrawing keto-group on the DPP core has an effect on the neighboring aromatic hydrogens, causing their signals to shift downfield. As a result, all nine molecules have two sets of doublets in the downfield 8.65-9.31 ppm, which are assigned to the $\beta-H$ on the thiophene rings linked to DPP core (Figure 1). The signals at $\sim 4.0$ ppm come from the $-\mathrm{NCH}_{2}$ - group on the DPP cores, and the signals at 7.28-7.3 ppm should be assigned to $\beta$-hydrogen on the terminated thiophene rings of the molecule chains. The downor upfield shifts of $\mathrm{Hc}$ and $\mathrm{Hd}$ of the molecules Ms1 9 are ascribed to the phenyl cores bearing various electron-withdrawing or electron-donating groups. Figure 1 describes the detailed analysis and assignments of the aromatic hydrogen of all molecules. 
The combined characterizations of ${ }^{1} \mathrm{H}$ and ${ }^{13} \mathrm{C}-\mathrm{NMR}, \mathrm{MALDI}-\mathrm{TOF}$ MS and elemental analysis clearly demonstrates that the molecules Ms1 9 have been successfully obtained. All the above spectra can be found in Supporting Information (SI). It is noteworthy that the nine synthesized molecules have good solubility in common organic solvents, such as $\mathrm{CH}_{2} \mathrm{Cl}_{2}$, toluene, $\mathrm{CHCl}_{3}$, and hexane, due to the presence of 2-hexyldecyl side chains. With these DPP-based D-A molecules (Ms1 9) in hand, we became interested in their molecular geometries and optical properties. The geometries of $\pi$-conjugated molecules were simulated by density functional theory (DFT) to understand the structure-property relationships. DFT calculations testified that the dihedral angle existed in the thiophene ring planes and phenyl ring planes (Figure S1). The molecules Ms1 5 have torsion due to the meta-substitution of phenyl rings by DPP. The skeletons of the molecules Ms1 5 exhibit a U-shaped appearance. DPP and benzene ring have a small torsion because of para-substitution of phenyl rings by DPP, strengthening the D-A interaction, which promotes the shift of $\pi$-electrons along the molecule backbones.

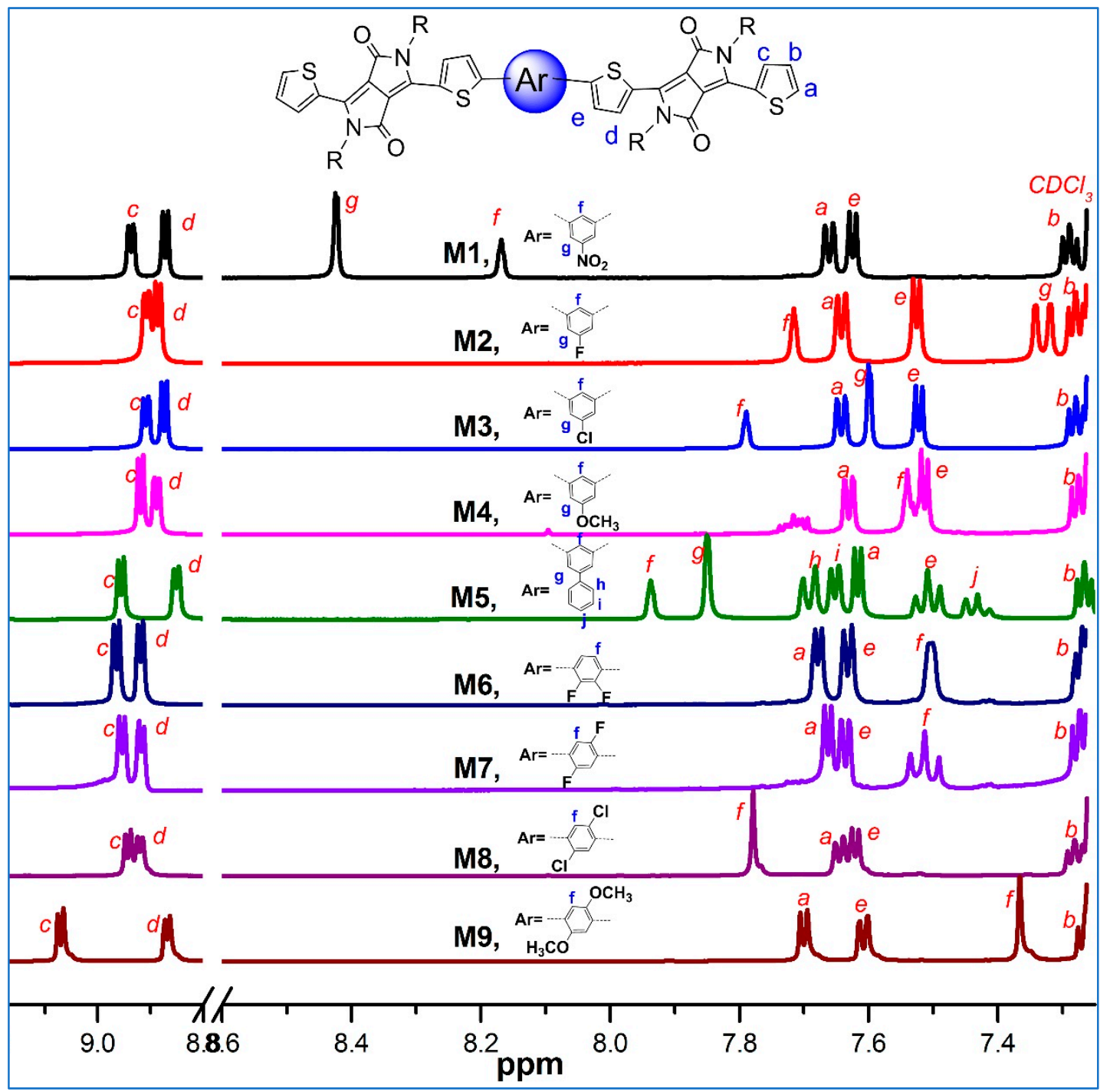

Figure 1. ${ }^{1} \mathrm{H}-\mathrm{NMR}$ spectra for Ms1 9 at 7.28-9.2 ppm.

As shown in Table 1, the phenyl cores of molecules bear various groups, including five electron-withdrawing groups, such as $-\mathrm{NO}_{2},-\mathrm{F}$, and $-\mathrm{Cl}$, an aryl group, and electron-donating group $-\mathrm{OCH}_{3}$. These groups, with their different electronegativity, should influence the optical and electrochemical properties of the $\pi$-conjugated molecules. The optical properties of the molecules Ms1 9 in solution and film were investigated using UV-vis spectroscopy. Figure 2 shows the UV-vis 
spectra of the molecules in $\mathrm{CHCl}_{3}$ solutions and films, and Table 2 summarizes the major optical properties, including light absorption peaks in $\mathrm{CHCl}_{3}$ solutions $\left(\lambda_{\max }{ }^{\mathrm{s}}\right)$ and solid films $\left(\lambda_{\max }{ }^{\mathrm{f}}\right)$ and corresponding absorption peak band-edges $\left(\lambda_{\text {onset }}^{\mathrm{s}}\right.$ and $\left.\lambda_{\text {onset }}{ }^{\mathrm{f}}\right)$. The molecules exhibited various colors in $\mathrm{CHCl}_{3}$, which corresponds to a wide range of light absorption between the visible and near-infrared regions. The light absorption peaks $\left(\lambda_{\max }\right)$ and band-edges $\left(\lambda_{\text {onset }}\right)$ and the energy levels are summarized in Table 1. As can be seen in Figure 2a and Table 2, the substituent groups, $-\mathrm{NO}_{2}$, $-\mathrm{F},-\mathrm{Cl},-\mathrm{OCH}_{3}$, and phenyl, have little influence on the light absorptions of meta-phenyl-cored DPP molecules Ms1 5, which showed almost the same trend in UV-vis spectra with little differences in $\lambda_{\max }$ s. The redshifts of $\lambda_{\max }$ s from $\mathbf{M} 1$ to $\mathbf{M} 2$ and $\mathbf{M} 1$ to $\mathbf{M} 3$, are 2 and $-1.5 \mathrm{~nm}$ (Table 2), respectively, which mean electron-withdrawing nitro groups on the $\pi$-conjugated molecules should provide the molecules with higher electron affinities. The small differences in $\lambda_{\max } \mathrm{s}$ among Ms1 5 should be attributed to the twisted geometries of meta-phenyl-cored DPP molecules, which caused a decreased conjugation and the disproportion between the repeating unit numbers and the effective conjugation lengths [36].
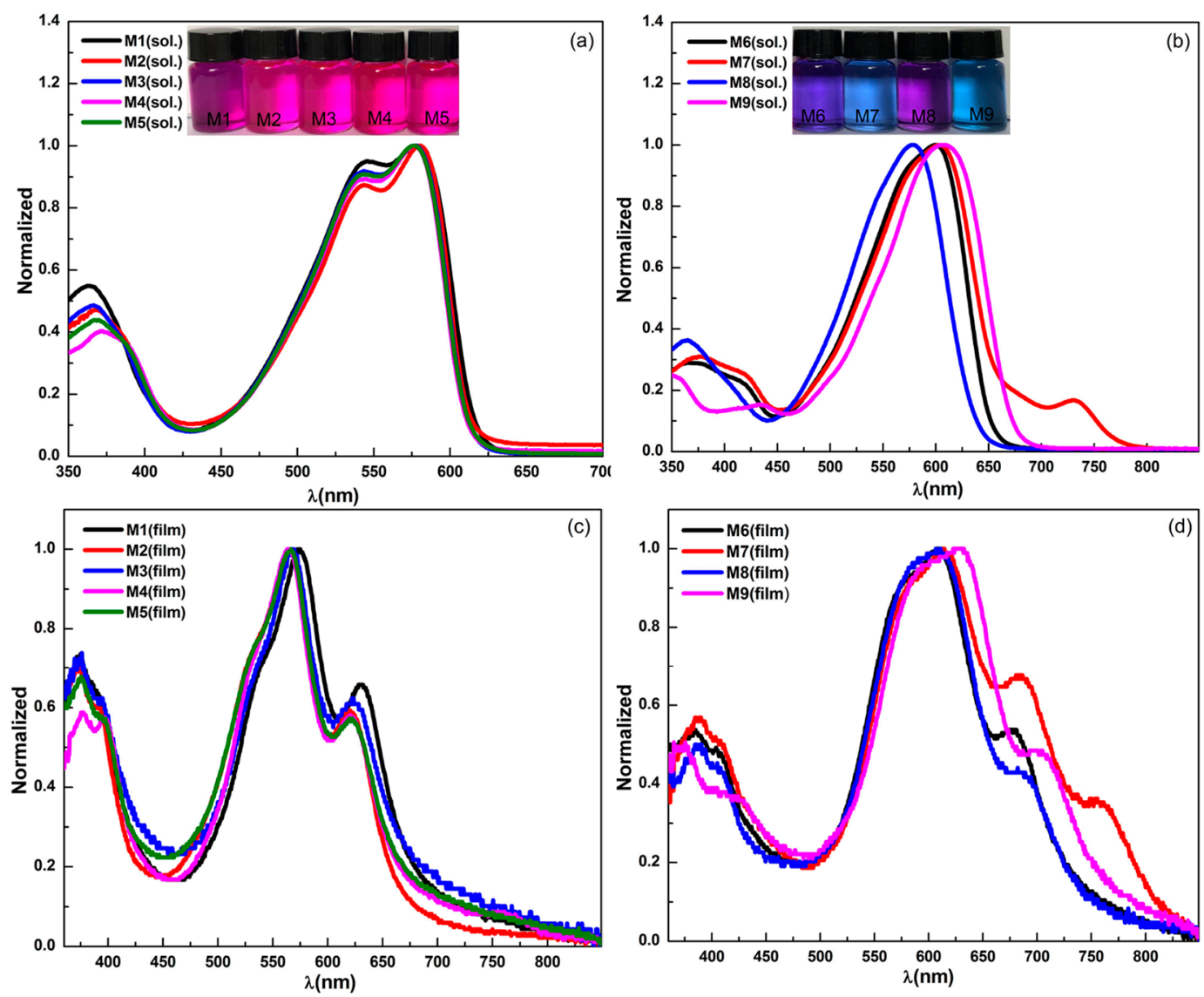

Figure 2. UV-vis spectra of meta-substituted phenyl-cored DPP molecules Ms1 5 and para-substituted phenyl-cored DPP molecules Ms6 9 (b) in $\mathrm{CHCl}_{3}(\mathbf{a}, \mathbf{b})$ and in films (c,d).

Compared with the meta-phenyl-cored DPP molecules Ms1 5, the substituent groups on the para-phenyl-cored DPP molecules Ms6 9 have larger influences on UV-vis absorption. The $\lambda_{\max } \mathrm{s}$ of Ms6 9 are 600, 603.5, 578.5, and $607 \mathrm{~nm}$, respectively, which shows larger differences between each molecule and various corresponding colors exhibited by the $\mathrm{CHCl}_{3}$ solutions of molecules Ms6 9 (Figure 2b). This might be due to the shortened conjugated length and the small contribution of groups in the meta-phenyl, thus, the enhanced delocalization of $\pi$-electron along the conjugated backbones and stronger D-A interaction between DPP and para-phenyl-cored molecules. The F and O atoms on M6, M7, and M9 have $\mathrm{F} \cdots \mathrm{H}$ or $\mathrm{O} \cdots \mathrm{H}$ noncovalent interactions with the $\mathrm{H}$ atoms on thiophene rings [37]. 
Besides the $\mathrm{O} \cdots \mathrm{H}$ noncovalent interaction, the molecule $\mathbf{M 9}$ also has $p-\pi$ conjugation between the $p$ orbital of $\mathrm{O}$ and the molecular backbone. As a result of synergic noncovalent interaction and $p-\pi$ conjugation, M9 possesses the longest wavelength of $\lambda_{\max }(607 \mathrm{~nm}$, Table 2) among the nine molecules. For molecule M8, the larger size of $\mathrm{Cl}$ atom and its steric effect should cause higher torsion and dihedral angle between the phenyl ring and thiophene ring, and thus decrease the delocalization of $\pi$-electron along the molecule backbone. Consequently, molecule M8 shows the shortest wavelength of $\lambda_{\max }$ among the para-phenyl-core molecules Ms6 9.

The UV-vis spectra for the solid films of molecules (Figure 2c,d) showed redshifts compared with their corresponding solutions because of their stronger intermolecular force, enhanced $\pi-\pi$ stacking, and thus effective intermolecular charge transfer. The absorption band-edges $\left(\lambda_{\text {onset }}\right)$ of the $\pi$-conjugated molecules and the corresponding optical bandgaps $\left(\mathrm{E}_{\mathrm{g}}{ }^{\mathrm{opt}}\right)$ calculated from $\mathrm{E}_{\mathrm{g}}{ }^{\mathrm{opt}}=$ 1240/ $\lambda_{\text {onset }}$ were also summarized in Table 2. The optical bandgaps of Ms1 5 and Ms6 9 are between 1.46 and $1.63 \mathrm{eV}$ (Table 2).

Table 1. One step synthesis of phenyl-cored DPP molecules (Ms1 9) ${ }^{1,2}$.
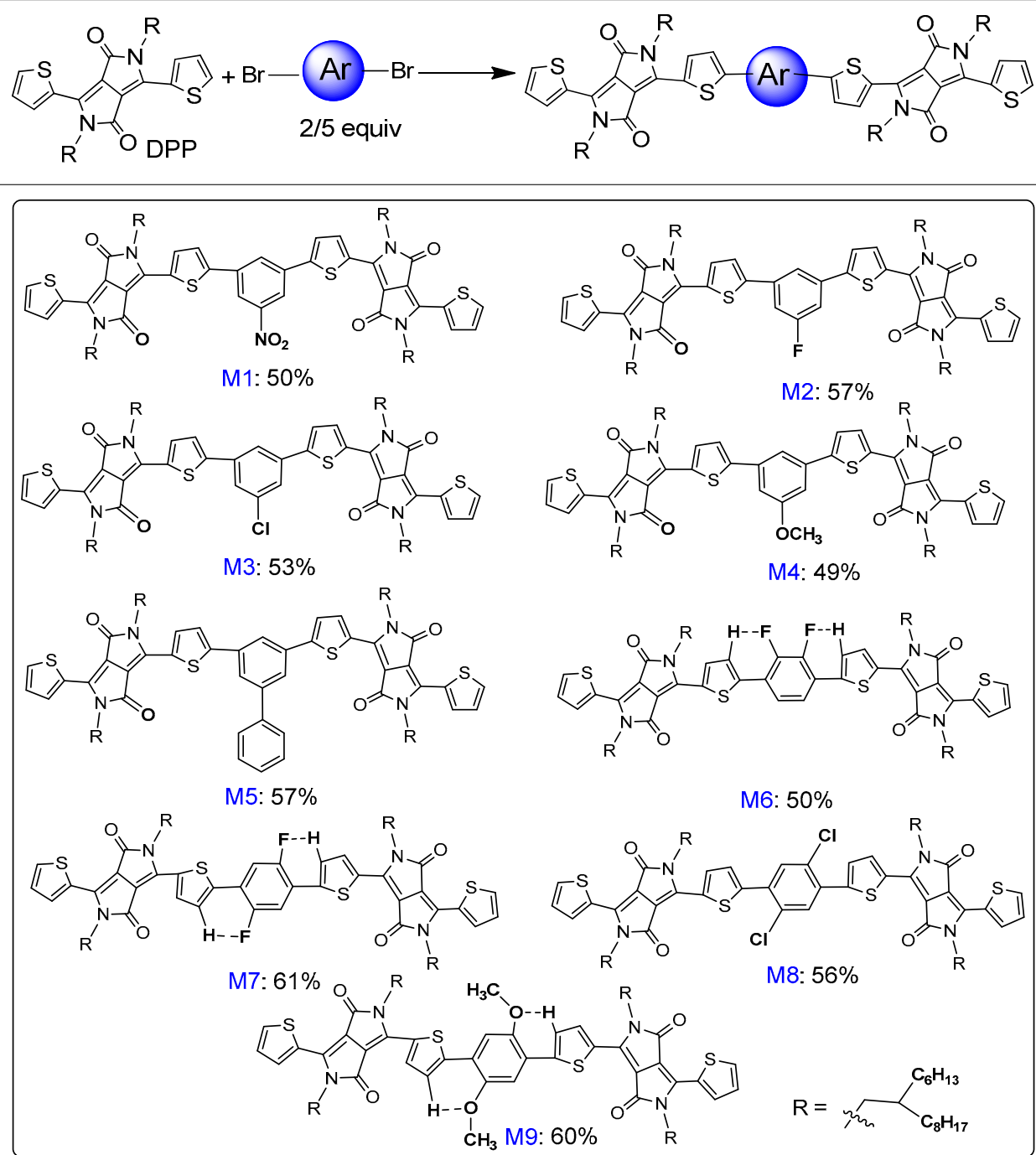

${ }^{1}$ All the reactions were conducted with TDPP $\left(200 \mathrm{mg}, 0.27 \mathrm{mmol}, 1\right.$ equiv), dibromoarene $\mathrm{R}^{\prime} \mathrm{ArBr}_{2}$ (2/5 equiv), $\mathrm{PivOH}(7.9 \mathrm{mg}, 0.08 \mathrm{mmol})$, anhydrous $\mathrm{Cs}_{2} \mathrm{CO}_{3}(200 \mathrm{mg}, 0.61 \mathrm{mmol}), \mathrm{Pd}_{2}(\mathrm{dba})_{3}(4.0 \mathrm{mg}, 1.5 \mathrm{~mol} \%)$, and $\mathrm{P}(\mathrm{o}-\mathrm{MeOPh})_{3}$ $(3.08 \mathrm{mg}, 3 \mathrm{~mol} \%)$ in $5 \mathrm{~mL}$ toluene at $100{ }^{\circ} \mathrm{C}$ for $24 \mathrm{~h}$ under argon atmosphere. ${ }^{2}$ Isolated yields. 
Table 2. Optical and electrochemical properties of molecules Ms1 9.

\begin{tabular}{cccccccc}
\hline Molecules & $\begin{array}{c}\lambda_{\max } \mathbf{s} \\
(\mathbf{n m})\end{array}$ & $\begin{array}{c}\lambda_{\text {max }} \mathbf{f} \\
(\mathbf{n m})\end{array}$ & $\begin{array}{c}\text { LUMO } \\
\mathbf{( e V )}\end{array}$ & $\begin{array}{c}\lambda_{\text {onset }} \mathbf{s} \\
(\mathbf{n m})\end{array}$ & $\begin{array}{c}\lambda_{\text {onset }} \mathbf{f} \\
(\mathbf{n m})\end{array}$ & $\begin{array}{c}\mathbf{E}_{\mathbf{g}} \text { opt } \\
(\mathbf{e V})\end{array}$ & $\begin{array}{c}\text { HOMO } \\
(\mathbf{e V})\end{array}$ \\
\hline M1 & 578.0 & 575.0 & -3.94 & 649 & 800 & 1.55 & -5.49 \\
M2 & 580.0 & 565.5 & -3.99 & 649 & 760 & 1.63 & -5.62 \\
M3 & 576.5 & 567.5 & -3.95 & 649 & 800 & 1.55 & -5.50 \\
M4 & 576.5 & 565.5 & -3.94 & 649 & 800 & 1.55 & -5.49 \\
M5 & 576.5 & 566.5 & -3.95 & 649 & 800 & 1.55 & -5.50 \\
M6 & 600.0 & 609.5 & -3.93 & 685 & 850 & 1.46 & -5.39 \\
M7 & 603.5 & 609.5 & -3.95 & 800 & 790 & 1.57 & -5.52 \\
M8 & 578.5 & 611.0 & -3.97 & 632 & 850 & 1.46 & -5.43 \\
M9 & 607.0 & 629.5 & -3.95 & 700 & 850 & 1.46 & -5.41 \\
\hline
\end{tabular}

The electrochemical characters of Ms1 9 were investigated by cyclic voltammetry (CV). The CV spectra of the nine DPPs are shown in Figure 3, and the measured frontier orbital energy (FMO) levels are summarized in Table 2. The lowest unoccupied molecular orbital (LUMO) energy levels were calculated based on the CV measurements, and the corresponding highest occupied molecular orbital $(\mathrm{HOMO})$ levels were calculated from $\mathrm{E}_{\mathrm{HOMO}}=\mathrm{E}_{\mathrm{LUMO}}-\mathrm{E}_{\mathrm{g}}{ }^{\mathrm{opt}}$. The LUMO levels of all molecules generally decreased with the increase of electron-withdrawing ability of the substitutions on phenyl cores. According to above UV-vis and CV measurements, most of the molecules might be used in middle bandgap D-A conjugated materials for device applications. Particularly, molecule M7 possesses deep HOMO levels and an extended absorption in the NIR region, which would promote its photoelectric properties for device applications.
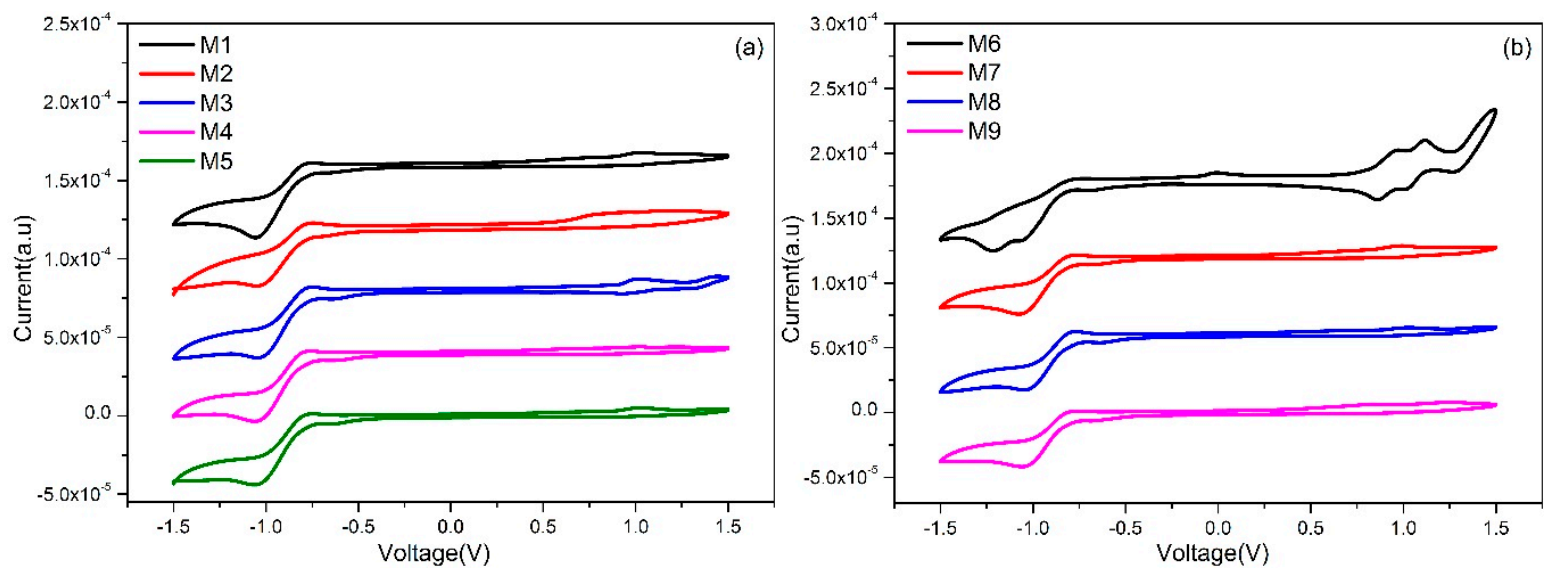

Figure 3. Cyclic voltammograms of meta-substitution phenyl-cored DPP molecules Ms1 5 (a) and para-substitution phenyl-cored DPP molecules Ms6 9 (b) in $\mathrm{CH}_{2} \mathrm{Cl}_{2}$.

\section{Materials and Methods}

\subsection{Materials}

Unless otherwise specified, all conventional chemicals were purchased from Energy Chemical (Shanghai, China). The starting TDPP was purchased from Derthon Co LTD (Shenzhen, China), 1,4-dibromo-2,5-difluorobenzene was purchased from SunaTech Inc (Suzhou, China). Anhydrous toluene was obtained by treating conventional toluene with $\mathrm{CaH}_{2}$.

\subsection{Characterizations}

All ${ }^{1} \mathrm{H}$ and ${ }^{13} \mathrm{C}$-NMR spectra were obtained in chloroform- $d$ or dichloromethane- $d$ using a Bruker Avance $400\left({ }^{1} \mathrm{H}-\mathrm{NMR} 400 \mathrm{MHz}\right.$ and ${ }^{13} \mathrm{C}-\mathrm{NMR} 101 \mathrm{MHz}$ ) spectrometer (Bruker, Germany). UV-vis absorption spectra were recorded on a Shimadzu UV-2450 spectrophotometer (Shimadzu Suzhou 
Instruments Mfg. Co. Ltd., Kyoto, Japan). Theoretical calculations based on DFT methods were performed for the molecules with Gaussian09 program Becke's three-parameter gradient-corrected functional (B3LYP) with 6-31G(d,p) basis for geometric optimization. Cyclic voltammetry (CV) was done on a CHI 660E electrochemical workstation (Hua Ke Putian Technology Co. Ltd., Beijing, China) with Pt disk, Pt plate, and standard 10 calomel electrode (SCE) as working electrode, counter electrode, and reference electrode, respectively, in a $0.1 \mathrm{~mol} / \mathrm{L}$ tetrabutylammonium hexafluorophosphate $\left(\mathrm{Bu}_{4} \mathrm{NPF}_{6}\right)$ $\mathrm{CH}_{2} \mathrm{Cl}_{2}$ solution, and the obtained $\mathrm{CV}$ curves were calibrated by recording the ferrocene-ferrocenium $\left(\mathrm{Fc} / \mathrm{Fc}^{+}\right)$redox couple $(4.8 \mathrm{eV})$ below the vacuum level versus the potential of the SCE.

\subsection{General Synthetic Procedure}

Typically, TDPP ( $200 \mathrm{mg}, 0.27 \mathrm{mmol}$ ), dibromobenzene derivatives $0.11 \mathrm{mmol}$, anhydrous $\mathrm{Cs}_{2} \mathrm{CO}_{3}$ ( $200 \mathrm{mg}, 0.61 \mathrm{mmol}), \mathrm{PivOH}(7.9 \mathrm{mg}, 0.08 \mathrm{mmol}), \mathrm{Pd}_{2}(\mathrm{dba})_{3}(4.00 \mathrm{mg}, 1.5 \mathrm{~mol} \%)$, tris(o-methoxyphenyl) phosphine $(3.08 \mathrm{mg}, 3 \mathrm{~mol} \%$ ) were successively added into a Schlenk tube. The tube was purged by three repetitions of vacuum and argon filling. Then, $5 \mathrm{~mL}$ anhydrous toluene was added via syringe. The reaction solution was deoxygenated using three freeze-vacuum-thaw cycles, and then rigorously stirred at $100{ }^{\circ} \mathrm{C}$ for $24 \mathrm{~h}$ under argon atmosphere. Removal of the toluene by rotary evaporator afforded the crude product, which was then purified by chromatogragh column (CC) on silica gel using a mixture of $\mathrm{CH}_{2} \mathrm{Cl}_{2}$ and hexane as eluent, giving the target molecules Ms1 9.

\subsection{Characterization Data of All Products}

6'-(5,5' -(5-Nitro-1,3-phenylene)bis(thiophene-5,2-diyl))bis(2,5-bis(2-hexyldecyl)-3-(thiophen-2-yl) pyrrolo[3,4-c]pyrrole-1,4(2H,5H)-dione) (M1): $95.53 \mathrm{mg}, 50 \%$ yield. ${ }^{1} \mathrm{H}-\mathrm{NMR}\left(400 \mathrm{MHz}, \mathrm{CDCl}_{3}\right) \delta 8.91$ $(\mathrm{dd}, J=26.3,3.9 \mathrm{~Hz}, 4 \mathrm{H}), 8.43(\mathrm{~d}, J=1.3 \mathrm{~Hz}, 2 \mathrm{H}), 8.17(\mathrm{~s}, 1 \mathrm{H}), 7.65(\mathrm{dd}, J=14.8,4.5 \mathrm{~Hz}, 4 \mathrm{H}), 7.29(\mathrm{dd}$, $J=9.0,4.2 \mathrm{~Hz}, 2 \mathrm{H}), 4.06(\mathrm{dd}, J=12.2,7.8 \mathrm{~Hz}, 8 \mathrm{H}), 2.07-1.87(\mathrm{~m}, 4 \mathrm{H}), 1.51-1.01(\mathrm{~m}, 96 \mathrm{H}), 0.83(\mathrm{dd}$, $J=18.3,5.5 \mathrm{~Hz}, 24 \mathrm{H}) .{ }^{13} \mathrm{C}-\mathrm{NMR}\left(101 \mathrm{MHz}, \mathrm{CDCl}_{3}\right) \delta 161.81,161.53,149.45,144.96,141.37,138.83,135.92$, 135.76, 131.01, 129.72, 128.53, 126.41, 120.05, 109.16, 108.03, 77.33, 77.02, 76.70, 46.34, 37.98, 37.76, 31.88, $31.85,31.76,31.34,31.22,30.04,30.01,29.70,29.67,29.52,29.50,29.29,26.33,22.66,22.62,14.10,14.07$, 14.03.MALDI-TOF MS (m/z): [M] ${ }^{+}$calcd for $\mathrm{C}_{98} \mathrm{H}_{145} \mathrm{~N}_{5} \mathrm{O}_{6} \mathrm{~S}_{4}: 1617.5070$, found 1617.0710. Elemental analysis: calcd for $\mathrm{C}_{98} \mathrm{H}_{145} \mathrm{~N}_{5} \mathrm{O}_{6} \mathrm{~S}_{4}, \mathrm{C}, 72.77 ; \mathrm{H}, 9.04 ; \mathrm{N}, 4.33 \%$. Found: $\mathrm{C}, 72.74 ; \mathrm{H}, 9.07 ; \mathrm{N}, 4.35 \%$.

6,6'-(5,5'-(5-fluoro-1,3-phenylene)bis(thiophene-5,2-diyl))bis(2,5-bis(2-hexyldecyl)-3-(thiophen-2-yl) pyrrolo[3,4-c]pyrrole-1,4(2H,5H)-dione) (M2): $103.0 \mathrm{mg}, 56.7 \%$ yield. ${ }^{1} \mathrm{H}-\mathrm{NMR}\left(400 \mathrm{MHz}, \mathrm{CDCl}_{3}\right) \delta$ $8.90(\mathrm{dd}, J=8.7,3.8 \mathrm{~Hz}, 4 \mathrm{H}), 7.72(\mathrm{~s}, 1 \mathrm{H}), 7.64(\mathrm{~d}, J=4.9 \mathrm{~Hz}, 2 \mathrm{H}), 7.53(\mathrm{~d}, J=4.0 \mathrm{~Hz}, 2 \mathrm{H}), 7.33(\mathrm{~d}$, $J=9.0 \mathrm{~Hz}, 2 \mathrm{H}), 7.31-7.27(\mathrm{~m}, 2 \mathrm{H}), 4.05(\mathrm{~s}, 8 \mathrm{H}), 1.94(\mathrm{~s}, 4 \mathrm{H}), 1.22(\mathrm{~s}, 96 \mathrm{H}), 0.99-0.74(\mathrm{~m}, 24 \mathrm{H}) .{ }^{13} \mathrm{C}-\mathrm{NMR}$ $\left(101 \mathrm{MHz}, \mathrm{CDCl}_{3}\right) \delta 161.75,146.79,140.80,136.18,135.46,130.72,130.02,129.82,128.46,125.61,108.73$, 77.33, 77.01, 76.69, 46.31, 37.95, 37.76, 31.88, 31.84, 31.79, 31.76, 31.36, 31.23, 30.05, 30.01, 29.71, 29.66, $29.54,29.49,29.29,26.35,26.23,22.62,14.09,14.06,14.02$. MALDI-TOF MS $(m / z):[\mathrm{M}]^{+}$calcd for $\mathrm{C}_{98} \mathrm{H}_{145} \mathrm{FN}_{4} \mathrm{O}_{4} \mathrm{~S}_{4}: 1590.5004$, found 1589.9700. Elemental analysis: calcd for $\mathrm{C}_{98} \mathrm{H}_{145} \mathrm{FN}_{4} \mathrm{O}_{4} \mathrm{~S}_{4}, \mathrm{C}, 74.01$; H, 9.19; N, 3.52\%. Found: C, 74.07; H, 9.22; N, 3.50\%.

6,6'-(5,5' -(5-chloro-1,3-phenylene)bis(thiophene-5,2-diyl))bis(2,5-bis(2-hexyldecyl)-3-(thiophen-2-yl) pyrrolo[3,4-c]pyrrole-1,4(2H,5H)-dione) (M3): $97.69 \mathrm{mg}$, $53.1 \%$ yield. ${ }^{1} \mathrm{H}-\mathrm{NMR}\left(400 \mathrm{MHz}, \mathrm{CDCl}_{3}\right) \delta$ 9.23-8.65 (m, 4H), $7.79(\mathrm{~s}, 1 \mathrm{H}), 7.74-7.62(\mathrm{~m}, 2 \mathrm{H}), 7.56(\mathrm{dd}, J=30.7,2.7 \mathrm{~Hz}, 4 \mathrm{H}), 7.31(\mathrm{dd}, J=26.6$, $22.6 \mathrm{~Hz}, 2 \mathrm{H}), 4.33-3.73(\mathrm{~m}, 8 \mathrm{H}), 1.97(\mathrm{t}, J=16.0 \mathrm{~Hz}, 4 \mathrm{H}), 1.50-1.05(\mathrm{~m}, 96 \mathrm{H}), 0.98-0.72(\mathrm{~m}, 24 \mathrm{H})$. ${ }^{13} \mathrm{C}-\mathrm{NMR}\left(101 \mathrm{MHz}, \mathrm{CDCl}_{3}\right) \delta 161.75,161.68,146.51,140.82,139.47,136.14,135.77,135.48,130.75$, 130.12, 129.81, 128.47, 125.64, 108.73, 77.33, 77.01, 76.70, 46.31, 37.95, 37.75, 31.88, 31.85, 31.80, 31.76, $31.23,30.06,30.01,29.30,26.40,26.23,26.19,22.63,14.08,-0.01$. MALDI-TOF MS $(\mathrm{m} / \mathrm{z})$ : $[\mathrm{M}]^{+}$calcd for $\mathrm{C}_{98} \mathrm{H}_{145} \mathrm{ClN}_{4} \mathrm{O}_{4} \mathrm{~S}_{4}$ : 1606.9520, found 1606.0140. Elemental analysis: calcd for $\mathrm{C}_{98} \mathrm{H}_{145} \mathrm{ClN}_{4} \mathrm{O}_{4} \mathrm{~S}_{4}, \mathrm{C}$, 73.25; H, 9.10; N, 3.49\%. Found: C, 73.26; H, 9.17; N, 3.53\%.

6,6'-(5,5'-(5-methoxy-1,3-phenylene)bis(thiophene-5,2-diyl))bis(2,5-bis(2-hexyldecyl)-3-(thiophen-2-yl) pyrrolo[3,4-c]pyrrole-1,4(2H,5H)-dione) (M4): $90.80 \mathrm{mg}, 49.5 \%$ yield. ${ }^{1} \mathrm{H}-\mathrm{NMR}\left(400 \mathrm{MHz}, \mathrm{CDCl}_{3}\right) \delta$ 
$8.91(\mathrm{dd}, J=12.5,4.0 \mathrm{~Hz}, 4 \mathrm{H}), 7.63(\mathrm{~d}, J=5.9 \mathrm{~Hz}, 2 \mathrm{H}), 7.57-7.45(\mathrm{~m}, 3 \mathrm{H}), 7.28(\mathrm{~d}, J=4.9 \mathrm{~Hz}, 2 \mathrm{H}), 7.17(\mathrm{~s}$, 2H), 4.09-3.93 (m, 8H), $1.96(\mathrm{~s}, 4 \mathrm{H}), 1.52-1.00(\mathrm{~m}, 96 \mathrm{H}), 0.94-0.76(\mathrm{~m}, 26 \mathrm{H}) .{ }^{13} \mathrm{C}-\mathrm{NMR}\left(101 \mathrm{MHz}, \mathrm{CDCl}_{3}\right)$ $\delta 161.73,148.43,140.43,139.94,136.44,135.41,135.26,129.88,129.41,128.42,125.20,108.42,77.33,77.01$, $76.69,55.60,46.31,37.92,37.76,31.88,31.79,31.76,31.35,31.23,30.39,30.07,30.01,29.73,29.54,29.50$, $29.29,28.94,27.74,26.36,26.20,23.78,22.98,22.62,19.16,14.07,14.03 . M A L D I-T O F$ MS $(\mathrm{m} / \mathrm{z}):[\mathrm{M}]^{+}$calcd for $\mathrm{C}_{99} \mathrm{H}_{148} \mathrm{~N}_{4} \mathrm{O}_{5} \mathrm{~S}_{4}$ : 1602.5360 , found 1602.0710. Elemental analysis: calcd for $\mathrm{C}_{99} \mathrm{H}_{148} \mathrm{~N}_{4} \mathrm{O}_{5} \mathrm{~S}_{4}, \mathrm{C}$, 74.20; H, 9.31; N, 3.50\%. Found: C, 74.59; H, 9.34; N, 3.46\%.

6,6'-(5,5' -([1,1'-biphenyl]-3,5-diyl)bis(thiophene-5,2-diyl))bis(2,5-bis(2-hexyldecyl)-3-(thiophen-2-yl) pyrrolo[3,4-c]pyrrole-1,4(2H,5H)-dione) (M5): $126.09 \mathrm{mg}$, 57.3\% yield. ${ }^{1} \mathrm{H}-\mathrm{NMR}\left(400 \mathrm{MHz}, \mathrm{CD}_{2} \mathrm{Cl}_{2}\right) \delta$ $8.98(\mathrm{~d}, J=4.1 \mathrm{~Hz}, 2 \mathrm{H}), 8.87(\mathrm{~d}, J=3.7 \mathrm{~Hz}, 2 \mathrm{H}), 7.96(\mathrm{~s}, 1 \mathrm{H}), 7.87(\mathrm{~s}, 2 \mathrm{H}), 7.71(\mathrm{~d}, J=7.4 \mathrm{~Hz}, 2 \mathrm{H}), 7.67(\mathrm{~d}$, $J=4.9 \mathrm{~Hz}, 2 \mathrm{H}), 7.64(\mathrm{~d}, J=4.1 \mathrm{~Hz}, 2 \mathrm{H}), 7.53(\mathrm{t}, J=7.5 \mathrm{~Hz}, 2 \mathrm{H}), 7.45(\mathrm{t}, J=7.3 \mathrm{~Hz}, 1 \mathrm{H}), 7.32-7.25(\mathrm{~m}$, $2 \mathrm{H}), 4.04(\mathrm{dd}, J=17.3,7.6 \mathrm{~Hz}, 8 \mathrm{H}), 1.93(\mathrm{~d}, J=26.9 \mathrm{~Hz}, 4 \mathrm{H}), 1.33-0.99(\mathrm{~m}, 96 \mathrm{H}), 0.88-0.76(\mathrm{~m}, 24 \mathrm{H})$. ${ }^{13} \mathrm{C}-\mathrm{NMR}\left(101 \mathrm{MHz}, \mathrm{CD}_{2} \mathrm{Cl}_{2}\right) \delta 161.57,139.69,136.38,134.95,129.02,128.22,127.18,125.12,68.33,53.94$, 53.67, 53.40, 53.13, 52.86, 46.11, 37.96, 37.77, 31.88, 31.84, 31.76, 31.31, 31.19, 30.04, 29.98, 29.65, 29.50, 29.30, 26.29, 26.16, 22.63, 13.84. MALDI-TOF MS (m/z): $[\mathrm{M}]^{+}$calcd for $\mathrm{C}_{104} \mathrm{H}_{150} \mathrm{~N}_{4} \mathrm{O}_{4} \mathrm{~S}_{4}$ : 1648.6080, found 1648.3150. Elemental analysis: calcd for $\mathrm{C}_{104} \mathrm{H}_{150} \mathrm{~N}_{4} \mathrm{O}_{4} \mathrm{~S}_{4}, \mathrm{C}, 75.77 ; \mathrm{H}, 9.17 ; \mathrm{N}, 3.40 \%$. Found: $\mathrm{C}$, $75.88 ; \mathrm{H}, 9.22 ; \mathrm{N}, 3.51 \%$.

6,6'-(5,5'-(2,3-difluoro-1,4-phenylene)bis(thiophene-5,2-diyl))bis(2,5-bis(2-hexyldecyl)-3-(thiophen-2-yl) pyrrolo[3,4-c]pyrrole-1,4(2H,5H)-dione) (M6): $92.15 \mathrm{mg}, 50.1 \%$ yield. ${ }^{1} \mathrm{H}-\mathrm{NMR}\left(400 \mathrm{MHz}, \mathrm{CDCl}_{3}\right) \delta$ $8.94(\mathrm{dd}, J=18.1,4.0 \mathrm{~Hz}, 4 \mathrm{H}), 7.68(\mathrm{~d}, J=4.1 \mathrm{~Hz}, 2 \mathrm{H}), 7.64(\mathrm{~d}, J=5.0 \mathrm{~Hz}, 2 \mathrm{H}), 7.50(\mathrm{~s}, 2 \mathrm{H}), 7.28(\mathrm{~d}$, $J=3.9 \mathrm{~Hz}, 2 \mathrm{H}), 4.05(\mathrm{dd}, J=12.8,7.7 \mathrm{~Hz}, 8 \mathrm{H}), 1.95(\mathrm{~s}, 4 \mathrm{H}), 1.51-1.07(\mathrm{~m}, 96 \mathrm{H}), 1.00-0.70(\mathrm{~m}, 24 \mathrm{H})$. ${ }^{13} \mathrm{C}-\mathrm{NMR}\left(101 \mathrm{MHz}, \mathrm{CDCl}_{3}\right) \delta 161.65,140.78,140.18,139.44,135.89,135.54,130.76,129.81,128.47$, $127.99,122.73,108.84,108.14,77.33,77.02,76.70,46.32,38.05,37.77,31.88,31.81,31.76,31.35,31.22$, $30.05,30.02,29.73,29.68,29.54,29.50,29.30,26.33,26.22,22.66,22.63,14.09,14.07,-0.01$.MALDI-TOF MS (m/z): [M] ${ }^{+}$calcd for $\mathrm{C}_{98} \mathrm{H}_{144} \mathrm{~F}_{2} \mathrm{~N}_{4} \mathrm{O}_{4} \mathrm{~S}_{4}: 1608.4900$, found 1608.2780. Elemental analysis: calcd for $\mathrm{C}_{98} \mathrm{H}_{144} \mathrm{~F}_{2} \mathrm{~N}_{4} \mathrm{O}_{4} \mathrm{~S}, \mathrm{C}, 73.18 ; \mathrm{H}, 9.02 ; \mathrm{N}, 3.48 \%$. Found: $\mathrm{C}, 73.25 ; \mathrm{H}, 9.08 ; \mathrm{N}, 3.52 \%$.

6,6'-(5,5'-(2,5-difluoro-1,4-phenylene)bis(thiophene-5,2-diyl))bis(2,5-bis(2-hexyldecyl)-3-(thiophen-2-yl) pyrrolo[3,4-c]pyrrole-1,4(2H,5H)-dione) (M7): $80.36 \mathrm{mg}, 60.6 \%$ yield. ${ }^{1} \mathrm{H}-\mathrm{NMR}\left(400 \mathrm{MHz}, \mathrm{CDCl}_{3}\right) \delta$ $8.94(\mathrm{dd}, J=14.9,4.0 \mathrm{~Hz}, 4 \mathrm{H}), 7.65(\mathrm{dd}, J=10.8,4.3 \mathrm{~Hz}, 4 \mathrm{H}), 7.52(\mathrm{t}, J=8.9 \mathrm{~Hz}, 2 \mathrm{H}), 7.28(\mathrm{~d}, J=4.8 \mathrm{~Hz}$, $2 \mathrm{H}), 4.05(\mathrm{dd}, J=12.4,7.7 \mathrm{~Hz}, 8 \mathrm{H}), 1.94(\mathrm{~s}, 4 \mathrm{H}), 1.49-1.05(\mathrm{~m}, 96 \mathrm{H}), 0.85(\mathrm{dd}, J=13.4,6.7 \mathrm{~Hz}, 24 \mathrm{H})$. ${ }^{13} \mathrm{C}-\mathrm{NMR}\left(101 \mathrm{MHz}, \mathrm{CDCl}_{3}\right) \delta 161.67,139.45,135.55,130.78,129.81,128.48,108.17,100.72,77.33,77.01$, 76.69, 46.34, 38.05, 37.76, 31.88, 31.79, 31.76, 31.35, 31.22, 30.05, 30.01, 29.72, 29.50, 29.29, 26.33, 26.19, 22.66, 22.62, 14.09, 14.06. MALDI-TOF MS (m/z): $[\mathrm{M}]^{+}$calcd for $\mathrm{C}_{98} \mathrm{H}_{144} \mathrm{~F}_{2} \mathrm{~N}_{4} \mathrm{O}_{4} \mathrm{~S}_{4}$ : 1608.4700, found 1608.6640. Elemental analysis: calcd for $\mathrm{C}_{98} \mathrm{H}_{144} \mathrm{~F}_{2} \mathrm{~N}_{4} \mathrm{O}_{4} \mathrm{~S}, \mathrm{C}, 73.18 ; \mathrm{H}, 9.02 ; \mathrm{N}, 3.48 \%$. Found: C, 73.29; $\mathrm{H}, 9.09 ; \mathrm{N}, 3.52 \%$.

6,6'-(5,5'-(2,5-dichloro-1,4-phenylene)bis(thiophene-5,2-diyl))bis(2,5-bis(2-hexyldecyl)-3-(thiophen-2-yl) pyrrolo[3,4-c]pyrrole-1,4(2H,5H)-dione) (M8): $104.87 \mathrm{mg}, 55.8 \%$ yield. ${ }^{1} \mathrm{H}-\mathrm{NMR}\left(400 \mathrm{MHz}, \mathrm{CDCl}_{3}\right) \delta$ $8.86(\mathrm{dd}, J=8.9,4.0 \mathrm{~Hz}, 4 \mathrm{H}), 7.71(\mathrm{~s}, 2 \mathrm{H}), 7.69-7.23(\mathrm{~m}, 4 \mathrm{H}), 7.21(\mathrm{~s}, 2 \mathrm{H}), 3.97(\mathrm{~d}, J=7.8 \mathrm{~Hz}, 8 \mathrm{H}), 1.88$ $(\mathrm{dd}, J=12.2,5.4 \mathrm{~Hz}, 4 \mathrm{H}), 1.40-0.97(\mathrm{~m}, 96 \mathrm{H}), 0.89-0.70(\mathrm{~m}, 24 \mathrm{H}) .{ }^{13} \mathrm{C}-\mathrm{NMR}\left(101 \mathrm{MHz}, \mathrm{CDCl}_{3}\right) \delta 160.77$, 160.66, 141.64, 139.88, 138.51, 134.52, 134.24, 131.68, 131.38, 130.44, 129.77, 129.66, 128.79, 128.51, 127.46, $107.81,107.11,76.32,76.00,75.68,45.31,37.06,36.75,30.87,30.77,30.31,30.22,29.05,29.00,28.69,28.66$, $28.51,28.28,25.32,25.21,25.18,21.65,21.62,13.08,13.06,0.00,-1.03 . M A L D I-T O F ~ M S ~(m / z): ~[M]^{+}$calcd for $\mathrm{C}_{98} \mathrm{H}_{144} \mathrm{Cl}_{2} \mathrm{~N}_{4} \mathrm{O}_{4} \mathrm{~S}_{4}: 1641.3800$, found 1641.6190. Elemental analysis: calcd for $\mathrm{C}_{98} \mathrm{H}_{144} \mathrm{Cl}_{2} \mathrm{~N}_{4} \mathrm{O}_{4} \mathrm{~S}$, C, $71.71 ; \mathrm{H}, 8.84 ; \mathrm{N}, 3.41 \%$. Found: $\mathrm{C}, 71.78 ; \mathrm{H}, 8.92 ; \mathrm{N}, 3.47 \%$.

6,6'-(5,5'-(2,5-dimethoxy-1,4-phenylene)bis(thiophene-5,2-diyl))bis(2,5-bis(2-hexyldecyl)-3-(thiophen2-yl)pyrrolo[3,4-c]pyrrole-1,4(2H,5H)-dione) (M9): $80.80 \mathrm{mg}, 59.7 \%$ yield. ${ }^{1} \mathrm{H}-\mathrm{NMR}\left(400 \mathrm{MHz}, \mathrm{CDCl}_{3}\right)$ $\delta 9.00(\mathrm{~d}, J=4.2 \mathrm{~Hz}, 2 \mathrm{H}), 8.80(\mathrm{~d}, J=3.8 \mathrm{~Hz}, 2 \mathrm{H}), 7.58(\mathrm{dd}, J=37.2,4.7 \mathrm{~Hz}, 4 \mathrm{H}), 7.45-7.19(\mathrm{~m}, 4 \mathrm{H}), 4.04$ $(\mathrm{d}, J=7.5 \mathrm{~Hz}, 6 \mathrm{H}), 3.97(\mathrm{~d}, J=4.1 \mathrm{~Hz}, 8 \mathrm{H}), 1.90(\mathrm{~d}, J=39.8 \mathrm{~Hz}, 4 \mathrm{H}), 1.40-0.97(\mathrm{~m}, 96 \mathrm{H}), 0.91-0.63(\mathrm{~m}$, 
24H). ${ }^{13} \mathrm{C}-\mathrm{NMR}\left(101 \mathrm{MHz}, \mathrm{CDCl}_{3}\right) \delta 161.69,150.37,139.70,135.86,130.20,126.40,111.27,108.37,77.32$, $77.01,76.69,56.40,31.88,31.76,31.24,30.12,29.68,29.51,29.30,26.21,22.64,14.08,1.02$. MALDI-TOF MS (m/z): $[\mathrm{M}]^{+}$calcd for $\mathrm{C}_{100} \mathrm{H}_{150} \mathrm{~N}_{4} \mathrm{O}_{6} \mathrm{~S}_{4}: 1632.5400$, found 1632.5570. Elemental analysis: calcd for $\mathrm{C}_{100} \mathrm{H}_{150} \mathrm{~N}_{4} \mathrm{O}_{6} \mathrm{~S}_{4}, \mathrm{C}, 73.57 ; \mathrm{H}, 9.26 ; \mathrm{N}, 3.43 \%$. Found: C, 73.59; H, 9.27; N, 3.48\%.

\section{Conclusions}

In summary, direct arylation of the $\mathrm{C}-\mathrm{H}$ bond has been demonstrated as an atom- and step-economic synthetic strategy for accessing DPP-based molecules. Nine DPP-based novel molecules Ms1 9, including five meta-phenyl-cored DPP molecules and four para-phenyl-cored DPP molecules, have all been synthesized in one step, in a facile manner, via direct $\mathrm{C}-\mathrm{H}$ arylation. All nine conjugated molecules, which had D-A structures and middle optical bands, have been well-characterized by NMR, MALDI-TOF MS, elemental analysis, cyclic voltammetry, and UV-vis absorption spectra. The current study would be a good reference for the atom- and step-economic synthesis of large DPP-based conjugated molecules.

Supplementary Materials: The following are available online at http://www.mdpi.com/1420-3049/24/9/1760/s1. Theoretical calculations based on DFT methods have been performed for the oligomers with Gaussian09 program: Figure S1; copies of ${ }^{1} \mathrm{H}-\mathrm{NMR}$ and ${ }^{13} \mathrm{C}-\mathrm{NMR}$ spectra of the products: Figures S2-S10; mass spectra of all oligomers: Figures S11-S19.

Author Contributions: H.L. synthesized the molecules, performed the NMR; X.-F.Z. and J.-Z.C. performed the CV and UV-vis. A.-G.Z. carried out the DFT calculations; H.-R.W. is the co-PI of H.L. and designed the molecules; S.-Y.L. is the co-PI of H.L. project administration, designed the molecules, and wrote the paper.

Funding: This research was funded by National Natural Science Foundation of China (Nos. 21374075, 21244008).

Acknowledgments: The authors thank Yu-Ping Wang for fruitful discussion.

Conflicts of Interest: The authors declare no conflict of interest.

\section{References}

1. Lin, Y.; Zhan, X. Oligomer Molecules for Efficient Organic Photovoltaics. Acc. Chem. Res. 2016, 49, $175-183$. [CrossRef] [PubMed]

2. Shirakawa, H.; Louis, E.J.; MacDiarmid, A.G.; Chiang, C.K.; Heeger, A.J. Synthesis of electrically conducting organic polymers: Halogen derivatives of polyacetylene, $(\mathrm{CH}) x$. J. Chem. Soc. Chem. Commun. 1977, 16, 578-580. [CrossRef]

3. Cai, Z.; Lo, W.-Y.; Zheng, T.; Li, L.; Zhang, N.; Hu, Y.; Yu, L. Exceptional Single-Molecule Transport Properties of Ladder-Type Heteroacene Molecular Wires. J. Am. Chem. Soc. 2016, 138, 10630-10635. [CrossRef] [PubMed]

4. Liu, S.Y.; Cheng, J.Z.; Zhang, X.-F.; Liu, H.; Shen, Z.-Q.; Wen, H.-R. Single-step access to a series of D-A $\pi$-conjugated oligomers with 3-10 nm chain lengths. Polym. Chem. 2019, 10, 325-330. [CrossRef]

5. Farnum, D.G.; Mehta, G.; Moore, G.G.I.; Siega, F.P. Attempted Reformatskii reaction of benzonitrile, 1,4-diketo-3,6-diphenylpyrrolo[3,4-C] pyrrole. A lactam analogue of pentalene. Tetrahedron Lett. 1974, 29, 2549-2552. [CrossRef]

6. He, C.-Y.; Wang, Z.; Wu, C.-Z.; Qing, F.-L.; Zhang, X. Pd-catalyzed oxidative cross-coupling between two electron-rich heteroarenes. Chem. Sci. 2013, 4, 3508-3513. [CrossRef]

7. Walker, B.; Tamayo, A.B.; Dang, X.D.; Zalar, P.; Seo, J.-H.; Garcia, A.; Tantiwiwat, M.; Nguyen, T.Q. Nanoscale Phase Separation and High Photovoltaic Efficiency in Solution-Processed, Small-Molecule Bulk Heterojunction Solar Cells. Adv. Funct. Mater. 2009, 19, 3063-3069. [CrossRef]

8. Pouliot, J.-R.; Sun, B.; Leduc, M.; Najari, A.; Li, Y.; Leclerc, M. A high mobility DPP-based polymer obtained via direct (hetero)arylation polymerization. Polym. Chem. 2015, 6, 278-282. [CrossRef]

9. Sonar, P.; Foong, T.R.B.; Dodabalapur, A. Synthesis of diketopyrrolopyrrole based copolymers via the direct arylation method for p-channel and ambipolar OFETs. Phys. Chem. Chem. Phys. 2014, 16, 4275-4283. [CrossRef] 
10. Bura, T.; Beaupré, S.; Légaré, M.-A.; Ibraikulov, O.A.; Leclerc, N.; Leclerc, M. Theoretical Calculations for Highly Selective Direct Heteroarylation Polymerization: New Nitrile-Substituted Dithienyl-Diketopyrrolopyrrole-Based Polymers. Molecules 2018, 23, 2324. [CrossRef]

11. Welsh, T.A.; Laventure, A.; Welch, G.C. Direct (Hetero)Arylation for the Synthesis of Molecular Materials: Coupling Thieno [3,4-c] pyrrole-4,6-dione with Perylene Diimide to Yield Novel Non-Fullerene Acceptors for Organic Solar Cells. Molecules 2018, 23, 931. [CrossRef] [PubMed]

12. Tang, H.; Xu, B.; Xiang, M.; Chen, X.; Wang, Y.; Liu, Z. Catalytic Performance of Nitrogen-Doped Activated Carbon Supported Pd Catalyst for Hydrodechlorination of 2,4-Dichlorophenol or Chloropentafluoroethane. Molecules 2019, 24, 4674. [CrossRef] [PubMed]

13. Tabasso, S.; Gaudino, E.C.; Acciardo, E.; Manzoli, M.; Giacomino, A.; Cravotto, G. Microwave-Assisted Dehydrogenative Cross Coupling Reactions in $\gamma$-valerolactone with a Reusable Pd/ $\beta$-cyclodextrin Crosslinked Catalyst. Molecules 2019, 24, 2288. [CrossRef] [PubMed]

14. Wakioka, M.; Yamashita, N.; Mori, H.; Nishihara, Y.; Ozawa, F. Synthesis of a 1,2-Dithienylethene-Containing Donor-Acceptor Polymer via Palladium-Catalyzed Direct Arylation Polymerization (DArP). Molecules 2018, 23, 4981. [CrossRef]

15. Obora, Y.; Ishii, Y. Pd(II)/HPMoV-Catalyzed Direct Oxidative Coupling Reaction of Benzenes with Olefins. Molecules 2010, 15, 1487-1500. [CrossRef] [PubMed]

16. Zhang, C.; You, L.; Chen, C. Palladium-Catalyzed C-H Arylation of 1,2,3-Triazoles. Molecules 2016, $21,1268$. [CrossRef]

17. Lafrance, M.; Rowley, C.N.; Woo, T.K.; Fagnou, K. Catalytic Intermolecular Direct Arylation of Perfluorobenzenes. J. Am. Chem. Soc. 2006, 128, 8754-8756. [CrossRef]

18. Lawrence, J.; Goto, E.; Ren, J.M.; McDearmon, B.; Kim, D.S.; Ochiai, Y. A Versatile and Efficient Strategy to Discrete Conjugated Oligomers. J. Am. Chem. Soc. 2017, 139, 13735-13739. [CrossRef]

19. Maria, F.D.; Olivelli, P.; Gazzano, M.; Zanelli, A.; Biasiucci, M.; Gigli, G. A Successful Chemical Strategy to Induce Oligothiophene Self-Assembly into Fibers with Tunable Shape and Function. J. Am. Chem. Soc. 2011, 133, 8654-8661. [CrossRef]

20. Wang, Q.; Qu, Y.; Tian, H.; Geng, Y.; Wang, F. Iterative Binomial Synthesis of Monodisperse Polyfluorenes up to 64-mers and Their Chain-Length-Dependent Properties. Macromolecules 2011, 44, 1256-1260. [CrossRef]

21. Burckstummer, H.; Weissenstein, A.; Bialas, D.; Wurthner, F. Colorimetric and Ratiometric Fluorescent Chemosensor Based on Diketopyrrolopyrrole for Selective Detection of Thiols: An Experimental and Theoretical Study. J. Org. Chem. 2011, 76, 2426-2432.

22. Kylberg, W.; Sonar, P.; Heier, J.; Tisserant, J.-N.; Muller, C.; Nuesch, F.; Chen, Z.-K.; Dodabalapur, A.; Yoon, S.; Hany, R. Synthesis, thin-film morphology, and comparative study of bulk and bilayer heterojunction organic photovoltaic devices using soluble diketopyrrolopyrrole molecules. Energy Environ. Sci. 2011, 4, 3617-3624. [CrossRef]

23. Chávez, P.; Bulut, I.; Fall, S.; Ibraikulov, O.A.; Chochos, C.L.; Bartringer, J.; Heiser, T.; Lévêque, P.; Leclerc, N. An Electron-Transporting Thiazole-Based Polymer Synthesized Through Direct (Hetero)Arylation Polymerization. Molecules 2018, 23, 1270. [CrossRef]

24. Kalepu, J.; Pilarski, T.L. Weinreb Amides as Directing Groups for Transition Metal-Catalyzed C-H Functionalizations. Molecules 2019, 24, 830. [CrossRef]

25. Pouliot, J.-R.; Grenier, F.; Blaskovits, J.T.; Beaupre, S.; Leclerc, M. Direct (Hetero)arylation Polymerization: Simplicity for Conjugated Polymer Synthesis. Chem. Rev. 2016, 116, 14225-14274. [CrossRef]

26. Josse, P.; Dayneko, S.; Zhang, Y.; Seignon, S.D.; Zhang, S.; Blanchard, P.; Welch, G.C.; Cabanetos, C. Direct (Hetero)Arylation Polymerization of a Spirobifluorene and a Dithienyl-Diketopyrrolopyrrole Derivative: New Donor Polymers for Organic Solar Cells. Molecules 2018, 23, 962. [CrossRef]

27. Nitti, A.; Po, R.; Bianchi, G.; Pasini, D. Direct Arylation Strategies in the Synthesis of $\pi$-Extended Monomers for Organic Polymeric Solar Cells. Molecules 2017, 22, 21. [CrossRef]

28. Liu, F.; Zhang, Y.; Wang, H.; Zhang, S. Novel Conjugated Polymers Prepared by Direct (Hetero) arylation: An Eco-Friendly Tool for Organic Electronics. Molecules 2018, 23, 408. [CrossRef]

29. Hendsbee, A.D.; Li, Y. Performance Comparisons of Polymer Semiconductors Synthesized by Direct (Hetero)Arylation Polymerization (DHAP) and Conventional Methods for Organic Thin Film Transistors and Organic Photovoltaics. Molecules 2018, 23, 1255. [CrossRef] 
30. Liu, S.-Y.; Shi, M.-M.; Huang, J.-C.; Jin, Z.-N.; Hu, X.-L.; Pan, J.-Y.; Li, H.-Y.; Jen, A.K.-Y.; Chen, H.-Z. C-H activation: Making diketopyrrolopyrrole derivatives easily accessible. J. Mater. Chem. A 2013, 1, 2795-2805. [CrossRef]

31. Zhang, J.; Kang, L.J.; Parker, T.C.; Blakey, S.B.; Luscombe, C.K.; Marder, S.R. Recent Developments in C-H Activation for Materials Science in the Center for Selective C-H Activation. Molecules 2018, 23, 922. [CrossRef]

32. Liu, S.-Y.; Liu, H.; Shen, Z.-Q.; Huang, W.-Y.; Zhong, A.-G.; Wen, H.R. Atom-and step-economic synthesis of $\pi$-conjugated large oligomers via C-H activated oligomerization. Dye. Pigment. 2019, 162, 640-646. [CrossRef]

33. Liu, S.-Y.; Wang, D.-G.; Zhong, A.-G.; Wen, H.-R. One-step rapid synthesis of $\pi$-conjugated large oligomers via C-H activation coupling. Org. Chem. Front. 2018, 5, 653-661. [CrossRef]

34. Lafrance, M.; Fagnou, K. Palladium-catalyzed benzene arylation: Incorporation of catalytic pivalic acid as a proton shuttle and a key element in catalyst design. J. Am. Chem. Soc. 2006, 128, 16496-16497. [CrossRef]

35. Lapointe, D.; Fagnou, K. Overview of the mechanistic work on the concerted metallation-deprotonation pathway. Chem. Lett. 2010, 39, 1118-1126. [CrossRef]

36. Sprick, R.S.; Bonillo, B.; Sachs, M.; Clowes, R.; Durrant, J.R.; Adamsa, D.J.; Cooper, I.A. Extended conjugated microporous polymers for photocatalytic hydrogen evolution from water. Chem. Commun. 2016, 52, 10008-10011. [CrossRef]

37. Li, S.; Zhan, L.; Liu, F.; Ren, J.; Shi, M.; Li, C.-Z.; Russell, T.P.; Chen, H. An Unfused-Core-Based Nonfullerene Acceptor Enables High-Efficiency Organic Solar Cells with Excellent Morphological Stability at High Temperatures. Adv. Mater. 2017, 30, 1705208. [CrossRef]

Sample Availability: Samples of the compounds Ms1 9 are all available from the authors.

(C) 2019 by the authors. Licensee MDPI, Basel, Switzerland. This article is an open access article distributed under the terms and conditions of the Creative Commons Attribution (CC BY) license (http://creativecommons.org/licenses/by/4.0/). 